\title{
Photon efficient wavefront sensing using an SLM for polarization-based weak measurements.
}

\author{
Mohammad Mirhosseini, ${ }^{1}$ Mehul Malik, ${ }^{1}$ Martin Lavery, ${ }^{2}$ Jonathan \\ Leach, ${ }^{3}$ Miles Padgett, ${ }^{2}$ and Robert W. Boyd ${ }^{1,3}$ \\ ${ }^{1}$ The Institute of Optics, University of Rochester, Rochester, NY 14627 USA \\ ${ }^{2}$ School of Physics and Astronomy, SUPA, Kelvin Building, University of \\ Glasgow, Glasgow G12 8QQ, Scotland, U.K. \\ ${ }^{3}$ Department of Physics, University of Ottawa, Ottawa ON K1N 6N5 Canada \\ moh.mir@gmail.com
}

\begin{abstract}
A novel scheme is proposed for two dimensional direct measurement of wave functions using an SLM. This photon efficient method can be potentially useful for wavefront sensing of turbulence in adaptive optics systems used in free-space communication systems.

(C) 2012 Optical Society of America

OCIS codes: ; (270.5585) Quantum information and processing;(010.7350) Wave-front sensing; (010.1330) Atmospheric turbulence;
\end{abstract}

Lundeen et al. [1] have recently proposed a method for direct measurement of the wave function of a photon using weak measurements. This method makes the measurement of the wave function possible through performing a weak measurement in position, using polarization as a pointer, and subsequently a strong measurements in momentum. It has also been pointed out that the same experiment can be alternatively described using classical optics [2]. In this picture interference of different portions of the same beam enables the measurement of the transverse profile of the electric field. All experiments previously done for the measurement of the wave functions using weak values, have been conducted only in one dimension. This limits the application of this technique to the cases where the input beams have uniform phase and amplitude in one direction. However this is a practical consequence of the approach chosen to perform the experiment and is not inherent to the theoretical framework. Here, we propose a variation of the experiment, modified by the use of a spatial light modulator (SLM) which can be used for measuring the full two-dimensional transverse amplitude and phase of an arbitrary input field. Applications of this technique include adaptive optics systems, optical metrology and measurements of aberrations. The single photon nature of this experiment also makes it potentially useful for applications with limitations in number of photons used for wavefront sensing. Adaptive optics systems used for mitigation of atmospheric turbulence in free space quantum communication systems are instances of this situation.

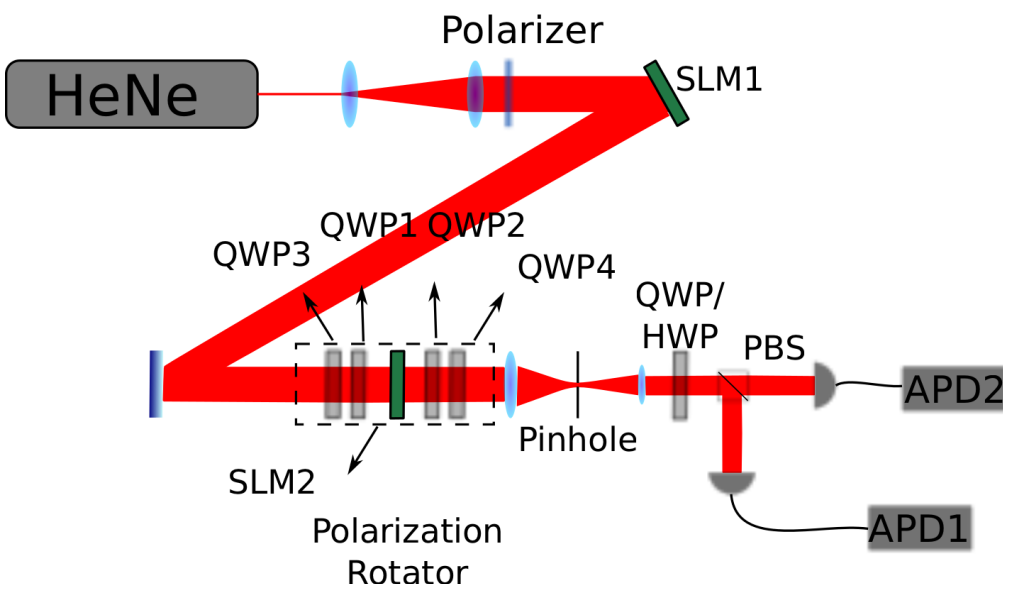

Fig. 1. Schematic of the unfolded experimental setup.

An ideal SLM can be modeled as a thin phase element. Such an element can be used with two quarter wave plates (QWP) to map phase retardance to polarization rotation [3]. The perfect phase plate model approximately describes a zero-twist SLM. However, most of the commercially available SLMs are twisted nematic liquid crystals(TNLCD), and have elliptical input and output eigen-polarizaton states, which makes their polarization behavior more complicated. It has been shown that TNLCDs can be used to achieve 
almost ideal polarization rotation using two additional QWPs [4]. Using this setup it is possible to change the polarization of any part of the input field by an arbitrary angle. The general spatial shape of the induced phase shift on the SLM also makes it possible to do the measurement in other bases rather than in the pixel basis. We have used this method to measure the aberrations induced by turbulence on a gaussian beam. The schematic of the setup can be found in Fig. 1. The first SLM is used to generate realizations of thin turbulence phase screens using Kolmogorov statistics. The resulting field is then projected on the second SLM, which along with the two QWPs before and after it, performs the polarization rotation. For measuring the phase and amplitude of the field at any point, we change the polarization of a small area which is centered at the point. The light from this part now interferes with the light from the rest of the beam to form a diffraction pattern after a Fourier transform lens. The $p=0$ component is then post selected using a pinhole. Analyzing the polarization component of the outputs provides the transverse amplitude and phase of the input field. This task is done using Diagonal/Anti-diagonal and Left-Handed/Right-Handed analyzers, a polarizing beam splitter(PBS) and two APDs.

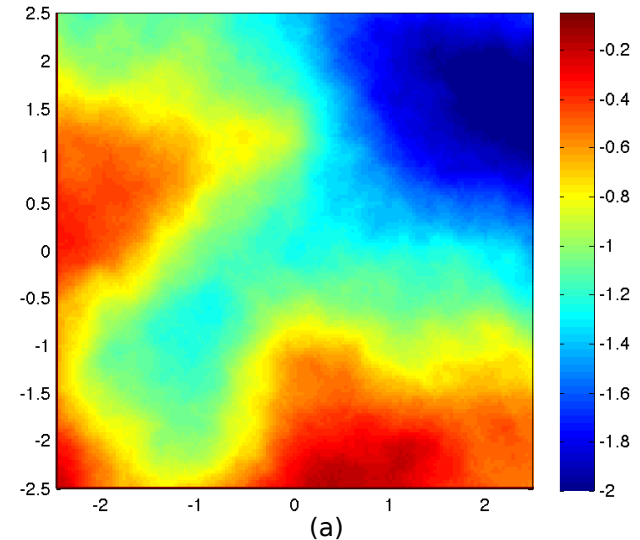

(a)

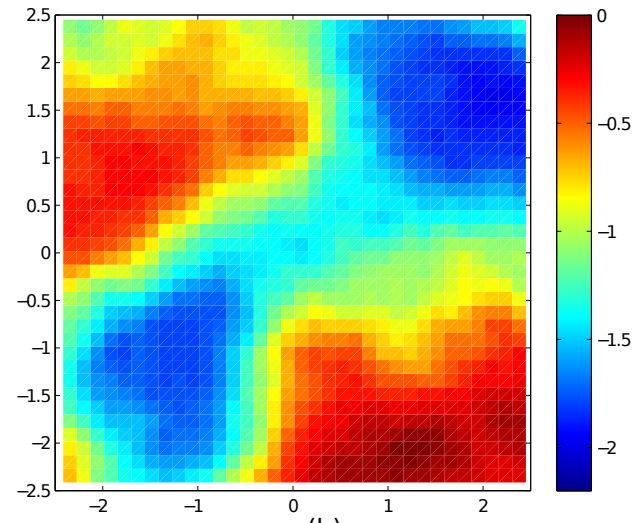

(b)

Fig. 2. Sample simulation of direct measurement of the wavefront of a plane wave propagated through atmospheric turbulence. Graphed are the (a) ideal phase of the beam from a realization of thin turbulence using Kolmogorov theory and (b) the phase resulted from the direct measurement of the wave function performed on the beam.

As a test of the experimental procedure, we have simulated the direct measurement of the wave front of a realization of thin turbulence and the results are presented in Fig 2. The simulated result from weak direct measurements are mostly consistent with the ideal values. (The difference between the ideal values and simulated results from the direct measurement are more pronounced when the measured phase approaches $-\pi / 2$, which is a numerical error commonly occurred where divisions by numbers very close to zero are performed). We hope to have experimental results to support these simulated results soon.

\section{References}

1. J. S. Lundeen, B. Sutherland, A. Patel, C. Stewart, and C. Bamber, "Direct measurement of the quantum wavefunction." Nature 474, 188-91 (2011).

2. C. Bamber, B. Sutherland, A. Patel, C. Stewart, and J. S. Lundeen, "Measurement of the transverse electric field profile of light by a self-referencing method with direct phase determination," Optics Express 20, 2034 (2012).

3. C. Ye, "Construction of an optical rotator using quarter-wave plates and an optical retarder," Optical Engineering 34, 3031 (1995).

4. I. Moreno, J. L. Martínez, and J. A. Davis, "Two-dimensional polarization rotator using a twisted-nematic liquid-crystal display," Applied Optics 46, 881 (2007). 